\title{
On the Deposition Rate of Magnetron Sputtered Thin Films at Oblique
}

\section{Angles}

R. Alvarez ${ }^{1 *}$, J.M. Garcia-Martin ${ }^{2}$, M.C. Lopez-Santos ${ }^{1}$, V.Rico ${ }^{1}$, F.J. Ferrer ${ }^{3}$, J. Cotrino ${ }^{1,4}$, A.R. Gonzalez-Elipe $^{1}$, A. Palmero ${ }^{1}$

1) Instituto de Ciencia de Materiales de Sevilla (CSIC-Universidad de Sevilla). c/ Americo Vespucio 49, 41092 Seville, Spain

2) IMM-Instituto de Microelectrónica de Madrid (CNM-CSIC). Isaac Newton 8, PTM, E-28760 Tres Cantos, Madrid, Spain.

3) Centro Nacional de Aceleradores (Universidad de Sevilla-CSIC-Junta de Andalucía), Thomas A. Edison 7, E-41092 Sevilla, Spain

4) Departamento de Física Atómica, Molecular y Nuclear, Universidad de Sevilla, Avenida Reina Mercedes s/n, E-41071 Seville, Spain

\section{Abstract}

We describe the magnetron sputtering deposition of thin films at oblique angles. A general relation between the deposition rate of the film and experimental parameters such as gas pressure or substrate tilt angle is deduced and experimentally tested. The model also permits the direct determination of the thermalization mean free path of the sputtered particles in the plasma gas, a key magnitude defining the balance between ballistic and diffusive flows in the deposition reactor. The good agreement between experimental and calculated results supports the validity of our description to explain the main features of the magnetron sputtering deposition of thin films at oblique angles.

*E-Mail: rafael.alvarez@icmse.csic.es 


\section{I.-Introduction}

Due to its stability, efficiency and up-scaling possibilities, magnetron sputtering (MS) deposition is nowadays one of the most popular techniques to grow thin films [1-4]. Although this deposition method has been traditionally employed to grow highly compact and flat materials, new microstructural possibilities are being reported in the last years when operating at oblique angles: in this case, shadowing-driven nanostructuration processes induce the development of highly porous structures, similar to those obtained in the absence of a plasma gas by e-beam assisted depositions in this geometry [5-12]. The MS technique, however, involves a higher degree of complexity associated to the presence of a plasma and to scattering events of sputtered particles in the gaseous phase. In general, much is known on the influence of experimentally controllable quantities on the thin film deposition, namely on the deposition rate, at classical (perpendicular) geometries [13-16]. This contrasts with the scarce knowledge available when operating at oblique angles, a fact that makes rather difficult the quantitative forecast of any deposition feature. In this paper, we develop a model to account for the variations of the MS deposition rate as a function of the gas pressure when operating the process at oblique angles. The influence on the deposition rate of significant experimental parameters (pressure, deposition angle) and related physical interaction events (scattering of the sputtered particles with the plasma gas) has been rationalized with a general formula whose validity has been tested against experimental data obtained for Ti thin films deposited by MS at oblique angles.

The transport of sputtered particles from the target towards the film is a problem long discussed in the literature since the late 70's and 80's. For instance in references [17, 18], the issues of the slowing down of sputtered particles when they move in a gas and their eventual thermalization were studied in detail by assuming a hard-sphere collisional dynamics, obtaining the energy distribution of the non-thermalized component of the particle flux as a distance to the target. More recently, the concept of "Effective Thermalizing Collision" (ETC) has been introduced in 
the literature in an attempt to simplify the description of the dynamics of sputtered atoms in the plasma gas [19-22]. In essence, it considers that each sputtered atom that leaves the target either follows straight trajectories up to its deposition onto a surface or it is scattered in the plasma gas by a single effective collision with plasma gas particles. This effective collision causes the loss of the original kinetic energy and momentum of the sputtered atoms that, by thermalization, will follow a thermal (random) motion within the plasma gas. A key magnitude in the ETC model is the so-called thermalization mean free path, $\lambda$, which accounts for the typical average distance covered by a sputtered atom in the plasma gas up to becoming thermalized. A so-called thermalization cross-section, $\sigma$, can be also defined through the relation $\lambda=1 / N \sigma$, where $N$ is the density of plasma gas particles: in reference [20], it was demonstrated that $\sigma$ is connected to the geometrical cross-section for an elastic scattering event between a sputtered atom and a plasma gas atom, $\sigma_{g}$, through the relation, $\sigma=\sigma_{g} / v$. As calculated by Westwood in reference [23], $v$ is the average number of subsequent elastic collisions required for the thermalization of the sputtered particles (i.e., for sputtered particles to acquire similar kinetic energy and momentum distribution of plasma gas particles). It is worth mentioning that inelastic collisions between heavy particles are neglected in this theory, an approximation that is associated to the low value of inelastic cross-sections in comparison with the elastic one in the typical energy range of sputtered particles (below $20 \mathrm{eV}$ ) [24].

The ETC approximation has successfully explained the so-called Keller-Simmons (K-S) formula, a well-known empirical equation in classical MS deposition (i.e., in a non-oblique configuration) with a single sputter gas [25]. The K-S formula illustrates the dependence between the deposition rate and relevant experimental parameters, such as the plasma gas pressure, $p_{g}$, and the distance between the cathode and the film, $L$. This formula reads 


$$
r_{K S}=\Phi_{0} \frac{p_{0} L_{0}}{p_{g} L}\left[1-\exp \left(-\frac{p_{g} L}{p_{0} L_{0}}\right)\right],
$$

where $r_{K S}$ is the deposition rate, $p_{0} L_{0}$ an adjustable parameter called characteristic pressuredistance product, and $\Phi_{0}$ is the flux of particles sputtered from the target. Regarding the sputtering theory, particles emitted from the target possess an energy spectrum proportional to $\Phi_{0} \propto E(E+U)^{-3} \cos \theta$, with $E$ the kinetic energy, $U$ the surface binding energy and $\theta$ the emission angle (see for instance ref. 16, 18). This implies that sputtered particles are highly directed in the direction perpendicular to the cathode with kinetic energies that peak around half the binding energy of the material, i.e., much above the typical thermal energies. In this way, equation (1) was deduced in ref. [19] from fundamental principles by assuming two main contributions to the film growth: i) the arrival of ballistic atoms which have preserved their original kinetic energy and directionality at the film surface, and ii) the arrival of sputtered atoms that have been thermalized by the plasma gas. Under these assumptions, the K-S formula was checked in one-dimensional description with $p_{0} L_{0} / p_{g} L=\lambda / L$ [20], by assuming that the temperature of the cathode, gas and film was equal.

Herein, we address the problem of the growth of thin films by MS at oblique angles, relating the deposition rate of the film with experimentally controllable process parameters. With this purpose, we have employed the ETC as a reference framework to describe the effect of the sputtered particles in different conditions, and developed a simple description of the deposition. It contrasts with other more sophisticated approaches in the literature concerning the transport of sputtered particles in the plasma gas, e.g., the SIMTRA code [26] or that in ref. [27], for instance. In this sense, our description implies a simple picture of the deposition that can be synthesized into a simple analytical formula that provides quantitative estimations on the film deposition rate without performing heavy computer calculations. In order to test this formula, we have compared its prediction with experimentally determined growth rates of Ti thin films, a 
test material very well studied under classical (non-oblique) conditions. The good agreement found between theoretical and experimental results ensures its validity.

\section{II.- Experimental Set-up}

The Ti thin films have been deposited by MS at different Ar pressures and deposition angles, using a $5 \mathrm{~cm}$ diameter Ti target. The base pressure of the chamber was in the mid $10^{-7} \mathrm{~Pa}$ range, being a $1 \mathrm{~cm}^{2}$ ultrasonically cleaned $\mathrm{Si}(100)$ substrate placed at $0.22 \mathrm{~m}$ from the target, and tilted by different angles with respect to its normal (see figure 1). The argon pressure was set at $0.15,0.5,1$ and $1.5 \mathrm{~Pa}$, whereas the tilt angle of the substrate, $\alpha$, was set at $0^{\circ}, 45^{\circ}, 60^{\circ}, 70^{\circ}, 80^{\circ}$ and $85^{\circ}$ for $p_{g}=0.15 \mathrm{~Pa}$, and $0^{\circ}, 45^{\circ}, 60^{\circ}$ at higher pressures. The DC electromagnetic generator was set at a constant power of $300 \mathrm{~W}$, indicating the plasma current and target potential in each experimental condition. The visible plasma glow covered a volume extending 50-75 $\mathrm{mm}$ from the target, i.e., always remaining several centimeters away from the substrate holder. The utilized magnetron head was supplied by AJA [28], and included a cylindrical metallic chimney placed next to the target $(5 \mathrm{~cm}$ radius and $9 \mathrm{~cm}$ length). This chimney is generically employed to avoid cross-contamination when other magnetron heads are within the deposition chamber, although it may introduce other collateral effects such as the partial collimation of the sputtered particle flux as well as the removal of sputtered atoms in the gaseous phase when deposited on its surface (see figure 1). The film temperature was always below $350 \mathrm{~K}$ during the sputtering process. Deposition times ranged from $90 \mathrm{~min}$ to $200 \mathrm{~min}$. Films were characterized by Rutherford Backscattering Spectroscopy (RBS) to assess the areal density. Experiments were carried out in a $3 \mathrm{MV}$ tandem accelerator at the Centro Nacional de Aceleradores (CNA, Seville, Spain) with a beam of $1.6 \mathrm{MeV}$ alpha particles, accumulated doses about $1.5 \mu \mathrm{C}, \sim 1 \mathrm{~mm}$ beam spot diameter, and a passivated implanted planar silicon (PIPS) detector located at $165^{\circ}$ scattering angle. The RBS spectra were simulated with the SIMRNA 
software [29], whereas the deposition rates were calculated by dividing the areal density of each film by the deposition time.

\section{III.- Description of the Deposition Process}

Following same philosophy as in ref. [19], we consider that the total deposition rate on the tilted substrate, $r_{\alpha}$, can be written as the sum of a highly directed and energetic ballistic contribution, $r_{B}$, and a diffusive contribution due to those sputtered atoms that have lost their original energy and move randomly in the plasma gas, $r_{D}$, i.e., $r_{\alpha}=r_{B}+r_{D}$. The ballistic contribution can be easily calculated by knowing that the flux of ballistic atoms at a distance $z$ from the target, $\Phi_{B}(z)$, is given by $\Phi_{B}(z)=\Phi_{0} \exp (-z / \lambda)$, where $\Phi_{0}$ is the flux of sputtered particles at the source position. Since the film is tilted an angle $\alpha$, the deposition rate of ballistic particles for $-90^{\circ} \leq \alpha \leq 90^{\circ}$, reads

$$
r_{B}=\Phi_{0} \exp \left(-\frac{L}{\lambda}\right) \cos \alpha
$$

(see figure 1 for more details). Next, we consider that, due to their non-preferential directionality in the plasma gas, the amount of thermalized atoms arriving at the film surface does not depend on the particular value of $\alpha$. Under this assumption, $r_{D}$ can be easily calculated for $\alpha=0$ as $r_{D}=r_{\alpha=0}-\left(r_{B}\right)_{\alpha=0}=r_{\alpha=0}-\Phi_{0} \exp (-L / \lambda)$, and thus, by introducing the quantity $\Delta r_{\alpha}=r_{\alpha=0}-r_{\alpha}$, we arrive at the formula,

$$
\Delta r_{\alpha}=\Phi_{0} \exp \left(-\frac{L}{\lambda}\right)(1-\cos \alpha)
$$


that relates the deposition rate with relevant process parameters such as $L / \lambda$, or the tilt angle of the film substrate. Furthermore, by knowing that $\lambda=1 / N \sigma$, with $N=p_{g} / k_{B} T_{g}$ being the density of gas particles ( $k_{B}$ is the Boltzmann constant and $T_{g}$ the gas temperature), and taking into account that, as explained above, $\sigma=\sigma_{g} / \nu$, the quantity $L / \lambda$ can be expressed as $L / \lambda=\chi p_{g} L$, with $\chi=\sigma_{g} / k_{B} T_{g} \nu$, and hence

$$
\Delta r_{\alpha}=\Phi_{0} \exp \left(-\chi p_{g} L\right)(1-\cos \alpha)
$$

Eq. (3) describes the dependence between the deposition rate and the tilt angle of the substrate, plasma gas pressure, target-film distance and the nature of the sputtered and gas atoms. It is worth to mention that the sole underlying hypotheses underpinning eq. (3) are that i) the ETC approximation applies and, ii) the flow of thermalized sputtered atoms arriving at the film surface does not depend on the value of $\alpha$. This means that no assumption has been made over diffusive fluxes in the deposition reactor, a feature that represents an essential difference in comparison with those utilized to deduce the K-S formula and that makes the present model more general. Therefore, eq. (3) would be applicable when the K-S formula is valid and the relation $r_{\alpha=0}=r_{K S}$ is fulfilled (with $p_{0} L_{0}=\chi^{-1}$ ) and, as we will see in section IV, in other situations where the K-S formula is not applicable. Interestingly, when $\alpha= \pm 90^{\circ}$, eq. (3) yields $r_{\alpha= \pm 90^{\circ}}=r_{\alpha=0}-\Phi_{0} \exp \left(-\chi p_{g} L\right)=r_{D}$, i.e. when the film is not facing the target, only diffusive flows contribute to the film grow. This agrees with previous results [30, 31], showing that $\mathrm{SiO}_{2}$ thin films deposited when the substrate was not facing the target grew at a much slower deposition rate by the sole incorporation of thermalized particles.

\section{IV.-Discussion and Experimental Validation}

In figure 2 we depict the determined deposition rates of the Ti thin films at a function of pressure in the classical configuration ( $\alpha=0)$. There, it is apparent that the deposition rate falls 
for higher background argon pressures, a feature that is linked with the increasing amount of thermalized sputtered particles and the decrease of the ballistic flux. Results in figure 2 contrast with those of Hartman [32] and Drüsedau [14], where they found that the K-S formula, eq. (1), was valid for MS depositions of Ti thin films with $p_{0} L_{0} \sim 13.8 \mathrm{~Pa} \mathrm{~cm}$ and $p_{0} L_{0} \sim 8 \mathrm{Pacm}$, respectively. Remarkably, the disagreement between these results in the literature and our data, depicted in figure 2, indicates the low reliability of the K-S formula in general conditions. By employing the software SIMTRA, a Monte Carlo model that describes the transport of sputtered particles from the target towards the substrate surface [26,33], it seems that the chimney is responsible for this difference, in particular, the removal of sputtered particles from the plasma gas by deposition on its surface. Indeed, SIMTRA assumes that once a sputtered particle reaches a surface within the reactor, it is removed from the discharge with a negligible backscattering or re-sputtering probability. This approximation that has been proven adequate for the deposition of Ti thin films [34]. As we will see next, the theory presented in this paper (section III) still holds in these conditions.

In figure 3 we have depicted the experimental values of $\Delta r_{\alpha}$ as a function of $\alpha$ for different deposition pressures. As expected, $\Delta r_{\alpha}$ increases for higher values of $\alpha$, and decreases with the deposition pressure. To determine the values of $\Delta r_{\alpha}$ from eq. (3) some basic calculations are required: the quantity $\chi$ is estimated by taking the following values: $T_{g} \sim 400 \mathrm{~K}$, $\sigma_{g}=\pi\left(R_{T i}+R_{A r}\right)^{2} \sqrt{1+\left(M_{T i} / M_{A r}\right)}=2.2 \times 10^{-19} \mathrm{~m}^{2} \quad\left(R_{T i}=147 \times 10^{-12} \mathrm{~m} \quad\right.$ and $R_{A r}=71 \times 10^{-12} \mathrm{~m}$ are the atomic radii of the $\mathrm{Ti}$ and $\mathrm{Ar}$ atoms, and $M_{T i}=47.9$ a.m.u., $M_{A r}=39.95$ a.m.u. their atomic masses, respectively), and $v \sim 3$, yielding $\chi=13.2 \mathrm{~Pa}^{-1} \mathrm{~m}^{-1}$. Regarding eq. (3), this value can be experimentally checked by representing $\ln \left(\Delta r_{\alpha}\right)$ as a function of $p_{g} L$, for a constant value of $\alpha>0^{\circ}$. This representation should yield a straight line defined by $\ln \left(\Delta r_{\alpha}\right)=a+b p_{g} L$ with $a=\ln \Phi_{0}+\ln (1-\cos \alpha)$ and $b=-\chi$. We have 
represented these quantities in figure 4 and obtained $\Phi_{0}=5.4 \times 10^{18}$ atoms $/ \mathrm{s} \mathrm{m}^{2}$ and $b=-12.3\left(\mathrm{~Pa}^{-1} \mathrm{~m}^{-1}\right)$ for $\alpha=45^{\circ}$, and $b=-13.2\left(\mathrm{~Pa}^{-1} \mathrm{~m}^{-1}\right)$ for $\alpha=60^{\circ}$, which remarkably match the calculated value, $\chi=13.2 \mathrm{~Pa}^{-1} \mathrm{~m}^{-1}$, deduced by our model. Hence, both theoretical and experimental results indicate that, under the analysed conditions, the calculated $\lambda$ values are $\lambda\left(p_{g}=0.15 \mathrm{~Pa}\right) \sim 50 \mathrm{~cm}, \quad \lambda\left(p_{g}=0.5 \mathrm{~Pa}\right) \sim 15 \mathrm{~cm}, \quad \lambda\left(p_{g}=1 \mathrm{~Pa}\right) \sim 7.5 \mathrm{~cm} \quad$ and $\lambda\left(p_{g}=1.5 \mathrm{~Pa}\right) \sim 5 \mathrm{~cm}$. To our knowledge, apart from the analysis performed in ref. [20], which presents indirect evidences about the validity of the ETC approach, this is the first time that a clear relation between $L / \lambda$ and $p_{g} L$ has been straightforwardly determined by proving a good match between experimental and theoretical values.

We have included in figure 3 the theoretical curves given by eq. (3), for $\Phi_{0}=5.4 \times 10^{18}$ atoms $/ \mathrm{s} \mathrm{m}^{2}$ and $\chi=13.2 \mathrm{~Pa}^{-1} \mathrm{~m}^{-1}$. In general, the agreement between our description and the experimental data is fairly good, particularly taking into account that the growth rates vary by more than two orders of magnitude under the studied conditions. This good concordance, together with the good match between calculated and experimental values of $\chi$, self-consistently ensure the validity of the ETC theory to describe the magnetron sputtering deposition of $\mathrm{Ti}$ thin films. In figure 2 we have included the calculated ballistic flux of deposited particles according to our model. These results together with those in figure 3 indicate that the depositions carried out at a pressure of $0.15 \mathrm{~Pa}(L / \lambda<1)$ are governed by ballistic flows and, therefore, the deposition rate shows a strong dependence on $\alpha$. Conversely, when the value of $L / \lambda$ increases (cases of $0.5 \mathrm{~Pa}, 1 \mathrm{~Pa}$ and $1.5 \mathrm{~Pa}$ ), the thermal component of the deposition flux becomes increasingly relevant and therefore the deposition rates show a weaker dependence on the substrate tilt angle. As a final remark, it is worth mentioning that although we have described the deposition under conditions where the K-S formula does not hold, the calculated value of $\chi=13.2 \mathrm{~Pa}^{-1} \mathrm{~m}^{-1}$ points towards a value of $p_{0} L_{0}=\chi^{-1}=7.7 \mathrm{~Pa} \mathrm{~cm}$ 
whenever it is applicable, in agreement with the reported values of $p_{0} L_{0}$ for MS deposition of Ti in absence of a chimney.

\section{V.- Conclusions}

In this paper we have deduced a simple equation that describes the deposition rate of thin films grown by magnetron sputtering at oblique angles. Through the value of $p_{g} L$ and the tilt angle of the substrate holder with respect to the target, this formula relates the deposition rate of thin films at oblique angles with that obtained in a classical (non-oblique) configuration. Besides, it permits a direct experimental estimation of the thermalization mean free path of sputtered particles. The model has been experimentally tested, finding a good agreement with experiments in all studied cases.

ACKNOWLEDGMENTS: We thank the Junta de Andalucía (Projects P09-CTS5189, TEP5283, P12-FQM-2265 and FQM-6900) and the Spanish MINECO (Projects CONSOLIDER CSD2008-00023, MAT2010-21228, MAT2010-18447, MAT201129194-C02-01) for financial support. 


\section{References}

[1] J. M. Schneider, S. Rohde, W. D. Sproul, and A. Matthews, J. Phys. D 33, 173(2000).

[2] K. Ellmer, T. Welzel, J. Mat. Res. 27(5), 765 (2012)

[3] I. Safi, Suf. Coat. Technol. 127(2-3) 203 (2000)

[4] P.J. Kelly, R.D. Arnell, VACUUM 56(3), 159 (2000)

[5] C.M. Zhou, D. Gall Appl. Phys. Lett. 88(20) 203117 (2006)

[6] S.V. Kesapragada, D. Gall, Appl. Phys. Lett. 89(20), 203121 (2006)

[7] D. Toledano, R.E. Galindo, M. Yuste, J.M. Albella, O. Sanchez, J. Phys. D: Appl. Phys. 46(4), $045306(2013)$

[8] F. Ruffino, M.G. Grimaldi, Nanosci. Nanotechnol. Lett. 4(3), 309 (2012)

[9] C. Patzig, T. Karabacak, B. Fuhrmann, B. Rauschenbach, J. Appl. Phys. 104(9), 094318 (2008)

[10] F.J. Garcia-Garcia, J. Gil-Rostra, A. Terriza, J.C. González, J. Cotrino, F. Frutos, F.J. Ferrer, A.R. González-Elipe, F. Yubero, Thin Solid Films, 542, 332 (2013)

[11] R Alvarez, J M García-Martín, M Macías-Montero, L Gonzalez-Garcia, J.C. González, V Rico, J Perlich, J Cotrino, A R González-Elipe, A Palmero Nanotechnology 24, 045604 (2013) [12] J. M. García-Martín, R. Alvarez, P. Romero-Gómez, A. Cebollada, A. Palmero, Appl. Phys. Lett. 97, 173103 (2010)

[13] T. P. Drüsedau, M. Lohmann, B. Garke, J. Vac. Sci. Technol. A 16, 2728 (1998).

[14] T. P. Drüsedau, Surf. Coat. Technol. 174-175, 470 (2003).

[15] T. Drüsedau, J. Vac. Sci. Technol. A 20, 459 (2002).

[16] Reactive Sputter Deposition, Springer Series in Materials Science, edited by D. Depla and S. Mahieu, ISBN 978-3-540-76662-9, Springer-Verlag Berlin Heidelberg 2008

[17] K. Meyer, I.K. Schuller, C.M. Falco, J. Appl. Phys. 52(9), 5803 (1981)

[18] A. Gras-Marti, J.A. Valles-Abarca, J. Appl. Phys. 54(2), 1071 (1983)

[19] A. Palmero, H. Rudolph and F. H. P. M. Habraken, Appl. Phys. Lett. 89, 211501 (2006) 
[20] A. Palmero, H. Rudolph and F. H. P. M. Habraken, J. Appl. Phys. 101, 083307 (2007)

[21] A. Palmero, H. Rudolph, F. H. P. M. Habraken, Appl. Phys. Lett. 87, 071501 (2005)

[22] A. Palmero, H. Rudolph, F.H.P.M. Habraken, Thin Solid Films, 515(2) 631, (2006)

[23] W. D. Westwood, J. Vac. Sci. Technol. 15, 1 (1978).

[24] Low Pressure Plasmas and Microstructuring Technology by Gerhard Franz, SpringerVerlag Berlin Heidelberg 2009, ISBN 978-3-540-85848-5, pages 28-30.

[25] J. H. Keller and R. G. Simmons, IBM J. Res. Dev. 23, 24 (1979)

[26] K. van Aeken, SIMTRA available at www.draft.ugent.be/

[27] F.J. Jimenez, S.K. Dew, J. Va. Sci. Technol. A 30, 041302 (2012)

[28] http://www.ajaint.com/

[29] M. Mayer, SIMNRA User's Guide, Tech. Rep. IPP 9/113, Max-Plank-Institut fur Plasmaphysik, Garching, Germany, 1997

[30] R. Alvarez, P. Romero-Gomez, J. Gil-Rostra, J. Cotrino, F. Yubero, A. Palmero, A. R. Gonzalez-Elipe, J. Appl. Phys. 108, 064316 (2010)

[31] R. Alvarez, P. Romero-Gomez, J. Gil-Rostra, J. Cotrino, F. Yubero, A. R. Gonzalez-Elipe, A. Palmero, Phys. Status Solidi A 210(4), 796 (2013)

[32] I. Knittel, M. Gothe, U. Hartmann, J. Vac. Sci. Technol. A 23(6), 1714 (2005)

[33] M. Horkel, K. Van Aeken, C. Eisenmenger-Sittner, D. Depla, S. Mahieu, W.P. Leroy, J. Phys. D: Appl. Phys. 43075302 (2010)

[34] T. Smy, R.V. Joshi, N. Tait, S.K. Dew and M.J. Brett, J. Appl. Phys. 84(9) 5315 (1998) 


\section{Figure Caption}

Figure 1.- Scheme of the main elements defining the theory

Figure 2.- Analysis of the deposition rate for $\alpha=0$. Experimental and ballistic data are in $3.5 \times 10^{18}$ atoms $/ \mathrm{s} \mathrm{m}^{2}$. Other data are in arbitrary units.

Figure 3.- Deposition rate dependence on the tilt angle of the substrate (lineal scale). Dots correspond to experimental data, whereas lines depict the theoretical prediction according to eq. (2). Note the different scales used for the low and high-pressure cases.

Figure 4.- Experimental determination of $\chi$. According with eq.(3), for a constant value of $\alpha$, $\Delta r_{\alpha}$ and $p_{g} L$ are linked through the relation $\ln \left(\Delta r_{\alpha}\right)=a+b p_{g} L$, with $a=\ln \Phi_{0}+\ln (1-\cos \alpha)$ and $b=-\chi$ 
Figure 1

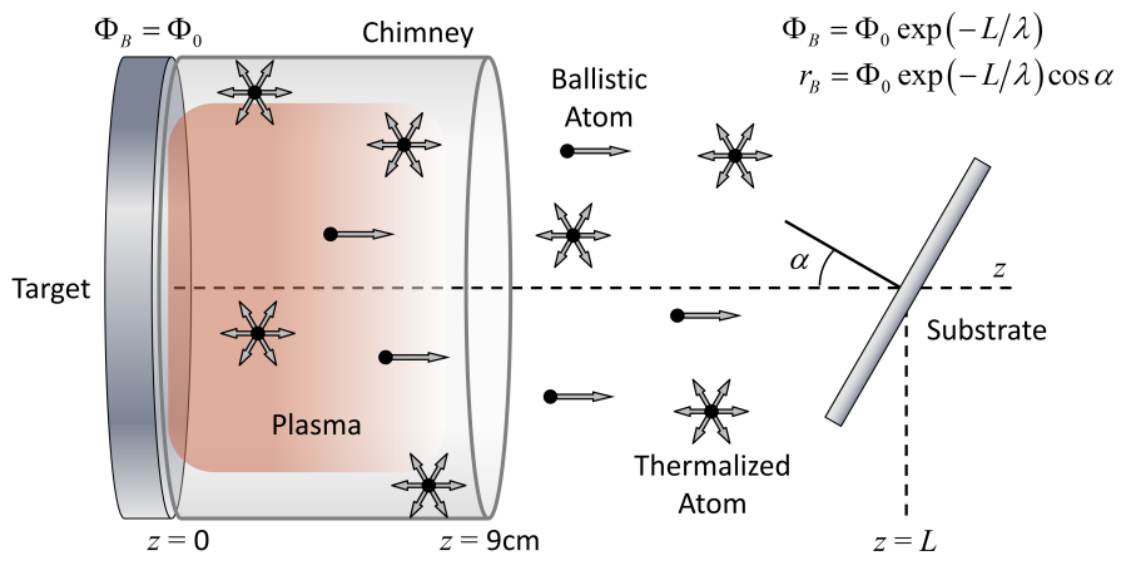


Figure 2

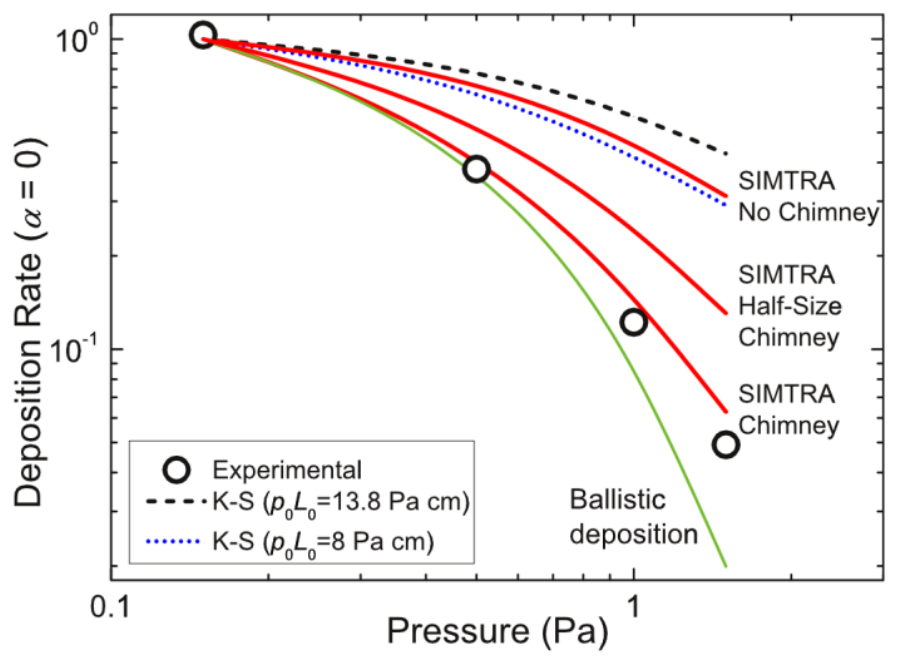


Figure 3

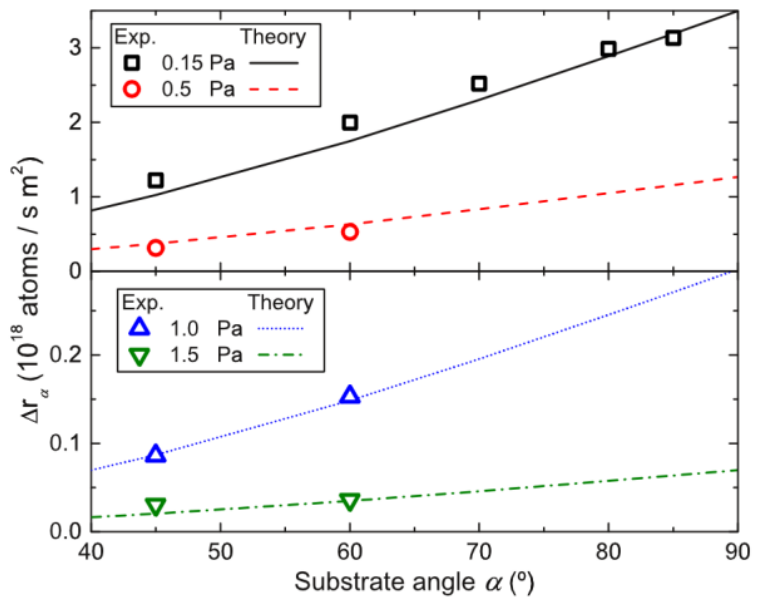


Figure 4

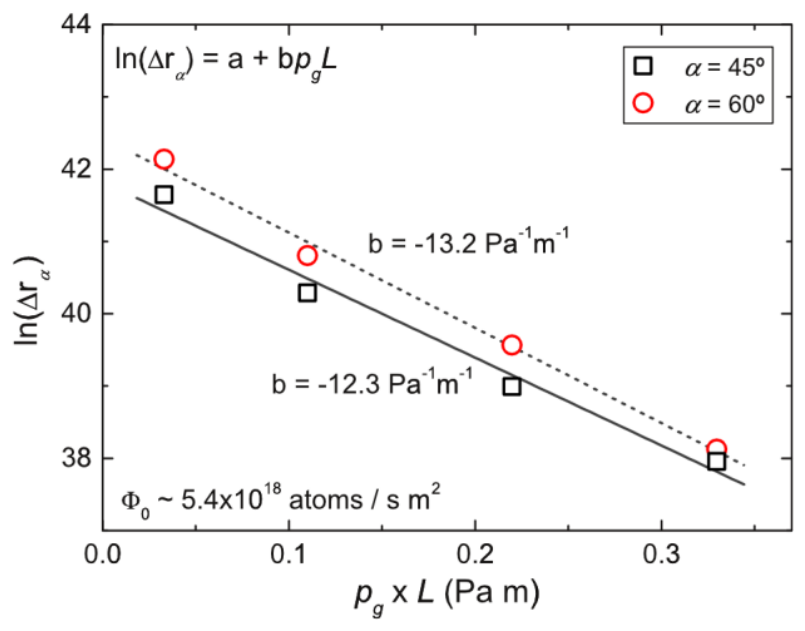

